\title{
Efecto de los factores protectores sobre los estilos de vida saludable en pacientes con riesgo cardiovascular: un análisis de ruta
}

\section{Effect of Protective Factors on Healthy Lifestyles in Patients with Cardiovascular Risk: Path Analysis \\ Efeito dos fatores protetores sobre os estilos de vida saudáveis em pacientes com risco cardiovascular: uma análise do caminho crítico}

\author{
Jesús Manuel Guerrero Alcedo** \\ Universidad Peruana de las Américas \\ Johanna Gabriela Sánchez Angulo \\ Universidad Nacional Autónoma de México \\ Víctor Manuel López Guerra \\ Universidad Técnica Particular de Loja
}

Doi: https://doi.org/10.12804/revistas.urosario.edu.co/apl/a.6233

\begin{abstract}
Resumen
El objetivo de la presente investigación fue verificar la influencia de factores individuales como la edad, el sexo, el nivel socioeconómico, y factores protectores como la resiliencia, la autoeficacia generalizada y el apoyo social percibido sobre los estilos de vida saludable, en personas con riesgo cardiovascular, además de sus posibles interacciones. Se utilizó una muestra de 290 personas, compuesta por 140 hombres y 150 mujeres, con edades comprendidas entre 35 y 65 años. A través de un análisis de ruta, se obtuvo que las variables con mayor predicción sobre el estilo de vida saludable fueron el
\end{abstract}

apoyo social percibido, específicamente de amigos, y la resiliencia. Así mismo, se encontró que la autoeficacia influye significativamente sobre la resiliencia, y el apoyo social percibido es un predictor relevante para la primera. En conclusión, el estilo de vida saludable se puede predecir en gran medida por variables de contenido psicológico, de forma directa e indirecta. Además, puede verse diferenciado dependiendo de la dimensión específica de la variable predicha y el número de variables que se relacionen en conjunto.

Palabras clave: factores protectores, hipertensión arterial, estilos de vida saludable, análisis de ruta.

* Este artículo es producto de una tesis de grado realizada por los dos primeros autores y tutoreada por el tercer autor en la Universidad Centroccidental Lisandro Alvarado, Venezuela. Dicho trabajo recibió el premio Ignacio Martín Baro Student Award, otorgado por la Sociedad Interamericana de Psicología.

** Dirigir correspondencia a Jesús Manuel Guerrero Alcedo: Escuela Profesional de Psicología, Facultad de Ciencias de la Comunicación y Psicología, Universidad Peruana de las Américas, Lima, Perú. Correo electrónico: jesusguerrero.psi@gmail.com

Para citar este artículo: Guerrero Alcedo, J. M., Sánchez Angulo, J. G., \& López Guerra, V. M. (2020) Efecto de los factores protectores sobre los estilos de vida saludable en pacientes con riesgo cardiovascular: un análisis de ruta. Avances en Psicología Latinoamericana, 38(1), 100-117. https://doi.org/10.12804/revistas.urosario.edu.co/apl/a.6233 


\section{flbstract}

The aim of this study was to verify the influence of individual factors such as age, sex, socioeconomic status, and protective factors like resilience, generalized self-efficacy, and social support perceived on healthy lifestyles in people with cardiovascular risk in addition to their possible interactions. We used a sample of 290 people, consisting of 140 men and 150 women, aged between 35 and 65 years. Through path analysis, we found that the most predictive variables on the healthy lifestyle were perceived social support, specifically friends' and resilience. We also found that self-efficacy significantly influences resilience, and perceived social support is a significant predictor of the former. In conclusion, the psychological content variables can predict a healthy lifestyle, directly and indirectly. It can also be differentiated depending on the specific dimension of the predicted variable and the number of variables that relate jointly.

Keywords: Protective factors, hypertension, healthy lifestyles, path analysis.

\section{Resumo}

$\mathrm{O}$ objetivo da presente pesquisa foi verificar a influência dos fatores individuais como a idade, o sexo, o nível socioeconômico e os fatores protetores como a resiliência, a auto-eficácia generalizada e o apoio social percebido sobre os estilos de vida saudável, em pessoas com risco cardiovascular, para além de suas possíveis interações. Utilizou-se uma amostra de 290 pessoas, composta por 140 homens e 150 mulheres, com idades compreendidas entre 35 e 65 anos. Através de uma análise do caminho crítico, obtivera-se que as variáveis com maior predição sobre o estilo de vida saudável foram o apoio social percebido, especificamente o apoio de amigos, e a resiliência. Igualmente, encontrou-se que a auto-eficácia inclui significativamente sobre a resiliência, e o apoio social percebido é um preditor significativo para a auto-eficácia. Em conclusão, o estilo de vida saudável pode-se predizer em grande medida por variáveis de conteúdo psicológico, de forma direta e indireta. Para além disso, pode se ver diferenciado dependendo da dimensão específica da variável predita e o número de variáveis que se relacionem em conjunto.

Palavras-chave: Fatores protetores, hipertensão arterial, estilos de vida saudável, análise do caminho crítico.

Las enfermedades cardiovasculares constituyen uno de los mayores problemas de salud pública en el mundo, ya que representan la primera causa de muerte en países industrializados, que está extendiéndose a los países en vías de desarrollo (Valdez et al., 2014; Veliz, Mendoza, \& Barriga, 2015). Venezuela no escapa de esta realidad, según las estadísticas del 2012, las enfermedades del corazón ocuparon el $20.58 \%$ de la mortalidad para ese año y en conjunto con las cerebrovasculares representan más de un $28.22 \%$ del total de las causas de muertes del país (Ministerio del Poder Popular para la Salud, 2014).

Diversos son los factores asociados a esta enfermedad, clasificados en no modificables tales como la edad, el sexo y el nivel socioeconómico, y modificables como hipertensión arterial, diabetes mellitus, sedentarismo, obesidad, tabaquismo, hipercolesterolemia y estrés, entre otros (López et al., 2014a, 2014b; Rojas et al., 2016). La hipertensión arterial es una de las principales causas de la enfermedad cardiovascular (Lira, 2015; Morales, Guillen, Muñoz, Belmar, Schifferli, Muñoz, \& Soto, 2017).

Actualmente se acepta que estos factores asociados al desarrollo de la enfermedad cardiovascular se encuentran relacionados a estilos o patrones de vida, los cuales determinan el proceso de salud-enfermedad (Córdoba et al., 2014; González, Llapur, Díaz, Illa, Yee, \& Pérez, 2015; Rojas et al., 2016).

Por este motivo, la aceptación de la idea que vincula los factores modificables con los estilos de vida saludable traería consigo una reorientación teórica en el campo de la salud, que se enfoca entonces en factores conductuales. De esta manera, desde el momento en el que se incorpora la conducta en el ámbito de la salud, la psicología cobra vigencia en el área (Rodríguez, 2016). 
Sin embargo, durante mucho tiempo el interés en psicología sobre este tema se ha centrado en el estudio de la patología, por lo que la modificación de estos estilos de vida o conductas relacionadas con las enfermedades cardiovasculares se ha direccionado bajo un enfoque de disminución de riesgos, dirigido a evitar la enfermedad y no a preservar la salud (Guerrero, \& Sánchez, 2015). Mientras se prevenga la enfermedad, la salud queda intacta, por ello resulta necesario reorientar las investigaciones hacia el extremo positivo de la salud, es decir, a sus factores de protección.

Es la psicología positiva, por iniciativa de Martin Seligman en 1999, la cual rescata y redirige un enfoque promotor de la salud, encaminado hacia el desarrollo de potencialidades de los seres humanos (Domínguez e Ibarra, 2017; Park, Peterson, \& Sun, 2013). Esta visión permite incorporar los factores positivos aportando una nueva pauta a la investigación, de manera que favorece el alcance de un mayor desarrollo psicológico, social y comunitario, que constituye una estrategia para el fomento de estilos de vida saludable (Vázquez, Hervás, Rahona, \& Gómez, 2009).

Es allí donde, basándose en este enfoque, se pretende redirigir el foco negativo del riesgo hacia el interés por las fortalezas humanas (Mariñelarena, 2016; Park et al., 2013), de manera que los factores promotores asociados a conductas saludables, como la autoeficacia (De León et al., 2016; Reigal, Videra, \& Gil, 2014), el apoyo social percibido (Arteaga, Collogo, \& Muñoz, 2017; Uribe, 2016) y la resiliencia (Antonio et al., 2015) ayuden a promocionar conductas dirigidas a metas. Además de estos factores psicológicos de protección, es necesario tener en cuenta aquellas variables individuales como edad, sexo y nivel socioeconómico que ayudan a conservar la salud.

Al igual que existen investigaciones que reportan factores de riesgo o patrones de vida asociados a las enfermedades cardiovasculares, también se han reportado elementos protectores psicológicos que contribuyen a la maximización de estos estilos de vida, resguardando y promoviendo la salud (Blanco, 2011).

Dentro de estos factores promotores de conductas saludables, se encuentra la edad, el sexo, el nivel socioeconómico, la autoeficacia generalizada, el apoyo social percibido y la resiliencia como una integración de varios elementos. Estas variables positivas han cambiado la forma como se percibe la salud del ser humano, pasando de un modelo de riesgo basado en la carencia, a un modelo de promoción dirigido a las fortalezas, para incrementar el nivel de bienestar. Este sería un gran aporte a la hora de promover factores de protección cardiovascular, aunque existan variables de riesgo como la hipertensión arterial (Acosta, \& Sánchez, 2009).

La autoeficacia es una propuesta basada en un modelo unificador que intenta explicar el cambio conductual, lo cual interpreta la motivación como una actividad que se manifiesta de manera espontánea, sin necesidad de reducirla a una reacción ante un estímulo especifico. Este término fue propuesto por Bandura (1977), quien lo define como el sentimiento de confianza en las propias capacidades para poder organizar y ejecutar las acciones necesarias para producir determinados logros o resultados. Las creencias de autoeficacia tienen lugar en la manera de pensar, sentir y actuar, por lo que si alguien ha desarrollado la capacidad de superar retos, pensará que puede hacerlo, se sentirá seguro, su motivación será más alta y llevará a cabo las acciones necesarias para lograrlo. De esta manera, es razonable pensar que dicha variable está relacionada directamente con los estilos de vida saludable, pues una mayor expectativa de autoeficacia lleva a la posibilidad de afrontar los estresores vitales, y a su vez, permite apreciar un estado de salud óptimo, así como llevar a cabo conductas más saludables, que protejan a las personas frente a la enfermedad.

El apoyo social percibido como variable relacionada a la salud centra su interés en la existencia de relaciones significativas y la evaluación subjetiva que realiza la persona sobre la adecuación 
del apoyo que recibe (Cuadra, Medina, \& Salazar, 2016). Su influencia en esta área se caracteriza por intervenir en la disminución del estrés, modulando la presión sanguínea, generando efectos sobre el sistema inmunológico y endocrino de las personas, y promoviendo conductas saludables como evitar consumir alcohol, drogas ilícitas, hacer dieta y realizar ejercicio, entre otros (Pacheco, Suárez, \& Angelucci, 1998). Algunos estudios refieren el efecto directo que tiene el apoyo social percibido sobre la salud física y psicológica (Howard, Creaven, Hughes, O’leary, \& James, 2017; Jung et al., 2016; Novoa, \& Barra, 2015), otros destacan el efecto moderador que tiene sobre el estrés (González, \& Estévez, 2017), y algunos autores manifiestan su relación indirecta sobre la salud, a través de otras variables como la resiliencia (Leiva, Pineda, \& Encina, 2013) y la autoeficacia (Miao, Feng, Wu, Zhang, Ge, \& Pan, 2015).

Por otra parte, la resiliencia se define como un "proceso dinámico que conlleva una adaptación positiva en contextos de gran adversidad" (Luthar, Cicchetti, \& Becker, 2000, p. 543). Desde esta perspectiva se reconocen en este constructo tres elementos clave que constituyen su definición: el proceso - no es una cualidad individual, sino que se da en interacción de mecanismos psicosociales-, la adversidad - antecedentes o desencadenante del comportamiento resiliente- y la adaptación positiva - éxito en la consecución de la conducta resiliente, que contaría con otras variables que coadyuvarían en su obtención- (García, García, López, \& Dias, 2016). Esta fortaleza relacionada al ámbito de la salud intenta dar cuenta de las situaciones de desarrollo saludable en presencia de factores de riesgo, que surge como un constructo teórico orientado a la promoción de habilidades que desarrollarán las personas resilientes para conseguir, mejorar y optimizar los recursos para el mantenimiento de la salud (García et al., 2016).

En el presente estudio se toma el apoyo social percibido y la autoeficacia generalizada, ya que se constituyen como factores de protección para la resiliencia, amortiguando los efectos de la adversidad y la vulnerabilidad, propiciando un aumento de la resiliencia, mayores conductas saludables y, por lo tanto, mejores resultados de salud (García et al., 2016).

A pesar de este enfoque esperanzador, los esfuerzos siguen dirigiéndose a la disminución del riesgo (Guerrero, 2014), y la enfermedad cardiovascular sigue siendo la principal causa de muerte (Escobar, \& Mariño, 2016); además, el direccionar las investigaciones solo al riesgo trae como consecuencia un vacío en los factores potenciadores de la salud. Sin embargo, considerar estos elementos positivos aislados tampoco sería suficiente; es necesario delimitar la conducta saludable como el resultado de múltiples variables individuales y sociales. Desde esta perspectiva, el análisis de ruta permite observar la interacción o la relación entre diversas variables hasta llegar a la ejecución de la conducta (Angelucci, 2007), por lo que sería factible destacar este método interaccionista en el contexto de los estilos de vida saludable, admitiendo no solo la visión integral del fenómeno, sino la verificación del modelo.

La importancia de la presente investigación no solo reside en el punto de vista teórico, sino en el práctico, donde al acceder a investigaciones con variables de protección y promoción del ser humano, se logren dos aportes importantes: guiar el camino hacia el extremo olvidado, como la promoción de la salud y no simplemente la prevención de la enfermedad, y dar pie al diseño e implementación de futuros programas que tomen en consideración factores protectores dentro del área cardiovascular.

Así pues, el objetivo de la presente investigación fue verificar la influencia de los factores individuales como la edad, el sexo, el nivel socioeconómico y los factores protectores como la resiliencia, la autoeficacia generalizada y el apoyo social percibido, sobre los estilos de vida saludable en personas con riesgo cardiovascular, además de sus posibles interacciones. 
Se plantea una hipótesis general que afirma que las variables personales como la edad, el sexo, el nivel socioeconómico y los factores protectores, como la autoeficacia generalizada, el apoyo social percibido y la resiliencia, inciden de manera directa o mediada sobre los estilos de vida saludables en personas con riesgo cardiovascular. Además, se plantean cuatro hipótesis específicas: 1) Una mayor edad, ser mujer, mayor nivel socioeconómico, alta percepción de apoyo social, autoeficacia generalizada elevada y resiliencia alta se asocian a un mayor estilo de vida saludable, 2) Mayor apoyo social percibido y autoeficacia generaliza elevada se asocian a mayor resiliencia, 3) Mayor apoyo social percibido se asocia a autoeficacia generaliza elevada, y 4) Menor edad y ser mujer se asocian a mayor apoyo social percibido.

\section{Método}

Se realizó un estudio de campo, no experimental de tipo correlacional, con un diseño de corte transversal y causal, a través de un análisis de ruta, el cual constituye una estrategia multivariada, donde se estiman relaciones directas e indirectas entre las variables, con el fin de encontrar la consistencia de los datos con las inferencias propuestas, cuyas bases radican en hallazgos válidos de investigaciones anteriores (Kerlinger, \& Lee, 2002).

\section{Participantes}

La selección de la muestra se hizo mediante un muestreo no probabilístico de tipo intencional. Esta estuvo conformada por 290 personas que asistieron al Centro Regional de Cardiología de la Región Centroccidental (Lara, Venezuela), 140 hombres y 150 mujeres en edades comprendidas entre 35 y 65 años, con diagnóstico médico de hipertensión arterial, sin otras patologías de comorbilidad.

\section{Instrumentos}

Escala de estilos de vida saludable: elaborada por Salazar y Arrivillaga (2004), fue adaptada a una población venezolana por Angelucci y Cañoto (2010). Esta escala consta de 60 ítems que reflejan 6 dimensiones: actividad física, recreación y manejo del tiempo libre, autocuidado y cuidado médico, hábito alimentario, consumo de alcohol u otras drogas y sueño. Las opciones de respuestas se basan en cuatro alternativas: nunca, algunas veces, frecuentemente o siempre, donde se deberá marcar con una $\mathrm{X}$ una de las cuatro opciones; los puntajes van desde 0 a 3 , donde el puntaje mínimo total es de 0 y el máximo de 180. Para el presente estudio, esta escala presenta un coeficiente alpha de Cronbach de 0.849 , que se correlaciona de manera positiva con el puntaje total de la prueba, lo que indica una alta consistencia interna. La validez de constructo fue determinada por análisis factorial exploratorio con rotación varimax, que arrojó una estructura factorial de nueve factores que explican el $44.70 \%$ de la varianza total explicada.

Escala de resiliencia: versión adaptada a Venezuela por Carratalá y Peña (2009), conformada por 23 ítems en forma de afirmación, en donde se identifican dos dimensiones: competencia personal y aceptación de uno mismo y de la vida; las respuestas son de tipo Likert, compuestas por siete opciones que van desde "totalmente en desacuerdo" hasta "totalmente de acuerdo". El mínimo puntaje es de 23 y el máximo posible de 161; las puntuaciones más altas representan un mayor nivel de resiliencia. En el presente estudio, la confiabilidad alpha de Cronbach arrojó un valor de 0.937 y la validez fue determinada bajo el análisis factorial exploratorio que arrojó una estructura unifactorial que explica el $45.56 \%$ de la varianza total explicada.

Escala de autoeficacia generalizada: la versión elaborada por Bäbler, Schwarzer y Jerusalem (1993) fue adaptada en una población chilena por Cid, Orellana y Barriga (2010), consta de 10 ítems, 
con un puntaje mínimo de 10 puntos y un máximo de 40 puntos. Las respuestas son tipo Likert donde la persona responde a cada reactivo de acuerdo con lo que ella percibe de su capacidad en el momento: incorrecto, apenas cierto, más bien cierto, cierto. La confiabilidad en la presenta investigación arrojó un alpha de Cronbach de 0.899 y la validez de criterio determinada por el análisis factorial exploratorio arrojó una estructura unidimensional que explica el $54.37 \%$ de la varianza total.

Escala de apoyo social percibido: adaptada a población venezolana por Angelucci (2001), se encuentra conformada por 28 ítems, en tres factores o dimensiones: apoyo de amigos y cercanos, apoyo familiar, y apoyo de grupos y comunidad religiosa. El formato de respuesta de la escala va desde "totalmente en desacuerdo" a "totalmente de acuerdo". A mayor puntaje en apoyo de amigos y cercanos, apoyo familiar y apoyo religioso, mayor apoyo social percibido. En la presente investigación, esta escala arrojó un alpha de Cronbach de 0.865 y una validez de constructo determinada bajo el análisis factorial exploratorio donde se identificaron tres factores que en conjunto explican el $39.64 \%$ de la varianza total explicada.

Escala de Graffar: permite obtener el puntaje total del nivel socioeconómico de la persona. Consta de cuatro ítems en forma de afirmación, cada una con cinco opciones de respuesta, que el participante deberá seleccionar con una X. Esta mide profesión del jefe de familia, profesión de la esposa(o) o cónyuge, principal fuente de ingreso y condiciones de alojamiento. Así, el puntaje va de 1 a 5 , donde 1 es el puntaje de la primera opción y 5 es el puntaje de la opción 5. A menor puntaje, mayor es el nivel socioeconómico de la persona (Méndez, 1994).

\section{Procedimiento}

Una vez obtenida la autorización del centro de salud, se procedió a solicitar el consentimiento o aprobación de cada una de las personas que consti- tuyeron la muestra estudiada, a través de un escrito, para luego originarse la aplicación de las escalas.

Posteriormente, se dieron las instrucciones para responder a cada uno de los instrumentos, de manera escrita y verbal. Durante el trascurso de la aplicación, el examinador estuvo presente para evitar consultas con otros participantes e interrupciones importantes al momento de responder el cuestionario. Se les hicieron las respectivas acotaciones a todos los sujetos: los resultados son confidenciales y de ninguna manera personas ajenas a la investigación podrán tener acceso a la información suministrada, esto con la finalidad de minimizar las respuestas sesgadas y asegurar mayor honestidad.

Por último, se corrigieron los instrumentos teniendo en consideración las especificaciones que ofrecen los autores de estas y se recopilaron los indicadores en una base de datos del programa SPSS 20.0 .

\section{Resultados}

Estadísticos descriptivos. Se procedió a calcular los estadísticos descriptivos con el objetivo de estudiar la distribución de los datos y el comportamiento de cada una de las variables implicadas en el diagrama de ruta (tabla 1).

En cuanto al estilo de vida saludable, se observa que los datos se distribuyen de forma uniforme y normal alrededor de la media con la misma cantidad de valores en ambos lados, las puntuaciones de la escala indican que los participantes presentan un estilo de vida saludable moderado.

Asimismo, se observan puntuaciones moderadas en la escala de resiliencia, que permiten constatar que la distribución de los datos tiende a variar ligeramente hacia los valores altos de la escala.

Por su parte, en la escala de autoeficacia generalizada, los participantes puntúan en su mayoría en el rango alto. La distribución tiende a orientarse hacia los valores altos de la escala, estos datos son moderadamente homogéneos. 
Con respecto al apoyo social percibido, se observan puntuaciones altas en la escala, lo cual indica que la mayoría de las personas del estudio presentan elevado apoyo social percibido.

Por último, los resultados de la escala del nivel socioeconómico indican que la mayoría de las personas tienen puntuaciones moderadas, lo cual muestra que el nivel socioeconómico al que pertenece la muestra es medio-bajo. Además, la distribución de los datos es normal y simétrica, con la misma cantidad de valores en ambos lados de la media.

En síntesis, la muestra estudiada se caracteriza por presentar una media de edad de 51 años, que se ubica en el nivel socioeconómico medio-bajo y muestra un moderado estilo de conductas saludables; en su mayoría son medianamente resilientes, con alto nivel de autoeficacia para llevar a cabo una conducta saludable. El apoyo social es de mucha importancia para dicho objetivo.

Análisis de correlaciones múltiples. Luego del análisis de los estadísticos descriptivos se procedió a calcular las relaciones planteadas en el diagrama de ruta (tabla 2). Para contrastar las hipótesis estadísticas planteadas y basándose en el tipo de investigación correlacional, se procedió a estimar el coeficiente de regresión estandarizado (Beta) mediante una técnica de cálculo de correlación simple o múltiple, dependiendo del número de variables predictores de cada variable endógena, usando para ello el método Producto Momento de Pearson. En cada relación planteada, se midió la varianza total explicada $\left(R^{2}\right)$ y el error o varianza no explicada (e), también llamadas medidas de bondad de ajuste, para ver el residuo y observar en qué medida la variación total de la variable $Y$ es explicada por el modelo de regresión.

Para utilizar este estadístico, fue necesario evaluar los supuestos básicos de multicolinealidad (tolerancia) y la autocorrelación según Durbin-Watson. Se obtuvo como resultado que no se viola ningún supuesto, debido a que la autocorrelación no excede el valor máximo de 2.5 , el valor de tolerancia que indica la multicolinealidad es $>0.10$ (Gardner, 2003).

A partir del cumplimiento de los supuestos de Pearson, se procede a verificar las relaciones planteadas a través de un análisis de regresión simple o múltiple, según el número de variables que afecten a la endógena, partiendo desde la derecha del diagrama, considerando coeficientes significativos aquellos puntajes obtenidos igual o menores a 0.05 .

Los resultados para la primera hipótesis presentados en la tabla 2, en cuanto a la relación planteada entre el estilo de vida saludable y las variables de resiliencia, autoeficacia generalizada, apoyo social percibido, sexo, edad y nivel socioeconómico arrojaron una correlación múltiple de 0.42 , lo cual indica una correlación positiva moderadamente

Tabla 1.

Estadísticos descriptivos de las variables implicadas en el modelo

\begin{tabular}{|c|c|c|c|c|c|c|c|c|}
\hline Variable & M & $\mathrm{Me}$ & DT & $\mathrm{CV}$ & AS & $\mathrm{K}$ & Min & Max \\
\hline Estilos de vida saludable & 114.39 & 115.00 & 17.967 & $16 \%$ & -0.059 & -0.273 & 71 & 162 \\
\hline Resiliencia & 130.94 & 138.00 & 22.937 & $18 \%$ & 1.564 & 2.008 & 28 & 161 \\
\hline Autoeficacia & 32.62 & 34.00 & 6.817 & $21 \%$ & -0.286 & 1.584 & 14 & 37 \\
\hline Apoyo social percibido & 57.08 & 59.00 & 13.630 & $24 \%$ & -0.799 & 0.401 & 14 & 84 \\
\hline Edad & 51.10 & 52.00 & 8.471 & $17 \%$ & -0.146 & -1.006 & 35 & 65 \\
\hline Nivel socioeconómico & 3.27 & 3.00 & 0.982 & $30 \%$ & -0.112 & -0.765 & 1 & 5 \\
\hline
\end{tabular}

Nota: $\mathrm{M}=$ media aritmética; $\mathrm{Me}=$ mediana; $\mathrm{DT}=$ desviación típica; $\mathrm{CV}=$ coeficiente de variación; $\mathrm{AS}=$ coeficiente de asimetría; $\mathrm{K}=$ coeficiente de curtosis; Min=valores mínimos, Max= valores máximos. 
alta entre estas variables, que explica de forma significativa el $16 \%$ de la varianza total del estilo de vida saludable por las variables predictoras implicadas en el modelo de regresión ( $R^{2}$ ajustada $=$ $0.16, F=10.205, p=0.000$ ).

Al evaluar la importancia por orden jerárquico de cada una de las variables predictoras sobre el estilo de vida saludable, los resultados arrojaron que las variables que la predicen significativamente fueron en primer lugar el apoyo social percibido $(\beta=0.272, p=0.000)$ y en segundo lugar la resiliencia $(\beta=0.166, p=0.010)$; es decir, que a mayor apoyo social y resiliencia, mayor fue el puntaje en la escala de estilo de vida saludable.

Con base en este resultado, y considerando que las variables que predicen fuertemente el estilo de vida saludable como la resiliencia y el apoyo social son bastantes amplias, se procede a realizar un análisis pormenorizado que permita estimar cuáles de las dimensiones de cada una de estas variables son las que ejercen mayor influencia sobre el estilo de vida saludable.

De esta manera, el apoyo social percibido arrojó que la dimensión que ejerce mayor influencia positiva es el apoyo percibido de amigos $(\beta=0.308$, $p=0.000)$. Esto indica que a mayor apoyo social percibido de amigos, mayor es el estilo de vida saludable; mientras que en relación a la resiliencia, al ver el peso de cada una de las dimensiones por separado sobre el estilo de vida saludable, se observó que no existe un peso significativo desde el punto de vista estadístico; es decir, la influencia sobre el estilo de vida saludable se observa en conjunto, pero no de forma separada.

Además, se observó que la influencia sobre el estilo de vida saludable se ve moderada también por el número de variables que se asocian a ella; de esta forma se observó que en las relaciones simples con el estilo de vida saludable resultaron significativas la autoeficacia con una relación positiva $(r=0.255, p=0.000)$ y el nivel socioeconómico de forma inversa $(r=-0.166, p=0.002)$, es decir, a mayor autoeficacia y menor nivel socioeconómico, mejor es el estilo de vida.

Por otra parte, en respuesta a la segunda hipótesis respecto a la relación entre la resiliencia y las variables de autoeficacia generalizada y apoyo social percibido, se observa un coeficiente de correlación de 0.53 , indicando que existe una relación positiva moderada entre las variables, que explica el $28 \%$ de la variación total de la resiliencia, por las variables predictoras de autoeficacia y apoyo social percibido, de forma significativa $\left(R^{2}=0.28\right.$, $F=22.325, p=0.000)$. Además, se encontró que la variable que predice mejor la resiliencia es la autoeficacia $(\beta=0.499, p=0.000)$, por lo que a mayor sentido de autoeficacia más alto es el nivel de resiliencia.

Como tercera hipótesis, la autoeficacia generalizada arrojó una relación positiva moderadamente baja con el apoyo social percibido, lo cual indica que la variación total de la autoeficacia es explicada en un $5 \%$ por esta variable $\left(R^{2}=\right.$ $0.05, F=16.164, p=0.000)$. De acuerdo con la importancia que tiene el apoyo social percibido sobre la autoeficacia generalizada se encontró que el coeficiente estandarizado fue significativo $(\beta=0.231, p=0.000)$. Esto indica que a mayor apoyo social percibido, mayor es la autoeficacia reportada en la escala.

Finalmente, en respuesta a la cuarta hipótesis, se observó que el apoyo social percibido al relacionarse con la edad y el sexo arroja un coeficiente de correlación múltiple de 0.098 , que indica una correlación positiva baja entre estas variables, $y$ explica que el apoyo social percibido varía totalmente en un $0.3 \%$ por la edad y el sexo, no es significativo desde el punto de vista estadístico $\left(R^{2}=0.003, F=1.396, p=0.249\right)$. Con respecto a la importancia de las variables sobre el apoyo social, no se encontraron relaciones significativas ni con la edad ni el sexo. 


\section{Jesús Manuel Guerrero flcedo, Johanna Gabriela Sánchez fngulo, Uíctor Manuel López Guerra}

Tabla 2.

Coeficientes de regresión para cada una de las hipótesis planteadas

\begin{tabular}{|c|c|c|c|c|c|c|c|c|}
\hline \multirow[t]{2}{*}{ Variables } & \multicolumn{4}{|c|}{ Correlación múltiple } & \multicolumn{2}{|c|}{$\begin{array}{l}\text { Coeficiente de } \\
\text { regresión }\end{array}$} & \multicolumn{2}{|c|}{ Correlación simple } \\
\hline & $\mathbf{R}$ & $\mathbf{R}^{2}(\mathbf{a})$ & $\mathbf{F}$ & $\mathbf{p}$ & $\beta(e)$ & p & $\mathbf{r}$ & p \\
\hline Estilos de vida saludable & 0.42 & 0.16 & 10.205 & $0.000 *$ & & & & \\
\hline Sexo & & & & & 0.009 & 0.873 & 0.048 & 0.414 \\
\hline Edad & & & & & 0.069 & 0.206 & 0.037 & 0.531 \\
\hline Nivel socioeconómico & & & & & -0.061 & 0.285 & -0.163 & $0.005^{*}$ \\
\hline Autoeficacia generalizada & & & & & 0.095 & 0.139 & 0.255 & $0.000 *$ \\
\hline Resiliencia & & & & & 0.166 & $0.010^{*}$ & & \\
\hline Apoyo social percibido & & & & & 0.272 & $0.000^{*}$ & & \\
\hline Amigos & & & & & 0.308 & $0.000^{*}$ & & \\
\hline Familia & & & & & 0.153 & 0.150 & & \\
\hline Grupo y comunidad & & & & & -0.051 & 0.449 & & \\
\hline Resiliencia & 0.53 & 0.28 & 22.325 & $0.000 *$ & & & & \\
\hline Autoeficacia generalizada & & & & & 0.499 & $0.000 *$ & & \\
\hline Apoyo social percibido & & & & & 0.097 & 0.060 & & \\
\hline Autoeficacia & 0.23 & 0.05 & 16.164 & $0.000^{*}$ & & & & \\
\hline Apoyo social percibido & & & & & 0.231 & $0.000^{*}$ & & \\
\hline Apoyo social percibido & 0.09 & 0.003 & 1.396 & 0.249 & & & & \\
\hline Sexo & & & & & 0.060 & 0.310 & & \\
\hline Edad & & & & & -1.350 & 0.178 & & \\
\hline
\end{tabular}

Nota: $* p<0.05 ; R=$ coeficiente de correlación múltiple; $R^{2}(a)=$ coeficiente de determinación múltiple ajustado; $F=$ prueba $F ; p=$ nivel de significancia; $\beta(e)=$ coeficientes de regresión parcial beta estandarizados; $r=$ coeficiente de correlación de Pearson.

En resumen, los resultados de la presente investigación confirman de forma parcial el modelo propuesto. Así pues, se encontró que el estilo de vida saludable se puede predecir en gran medida por el apoyo social percibido, específicamente por el apoyo de amigos, y en segundo lugar por la resiliencia. Por su parte, el nivel de resiliencia está influido por la autoeficacia generalizada y esta última se determina significativamente a partir del apoyo social percibido.

En definitiva, el peso de la influencia de cada una de las variables predictoras del estilo de vida saludable puede verse diferenciado dependiendo de la dimensión específica de la variable predicha, además de relacionarse a otras variables de forma indirecta. En este caso, esto resulta en una relación directa y significativa del apoyo social percibido de amigos sobre el estilo de vida saludable, además de funcionar como predictor indirecto sobre ella, la autoeficacia (figura 1).

\section{Discusión}

Esta investigación tiene como objetivo verificar empíricamente a través de un análisis de ruta la influencia de los factores individuales como la edad, el sexo, el nivel socioeconómico y los 


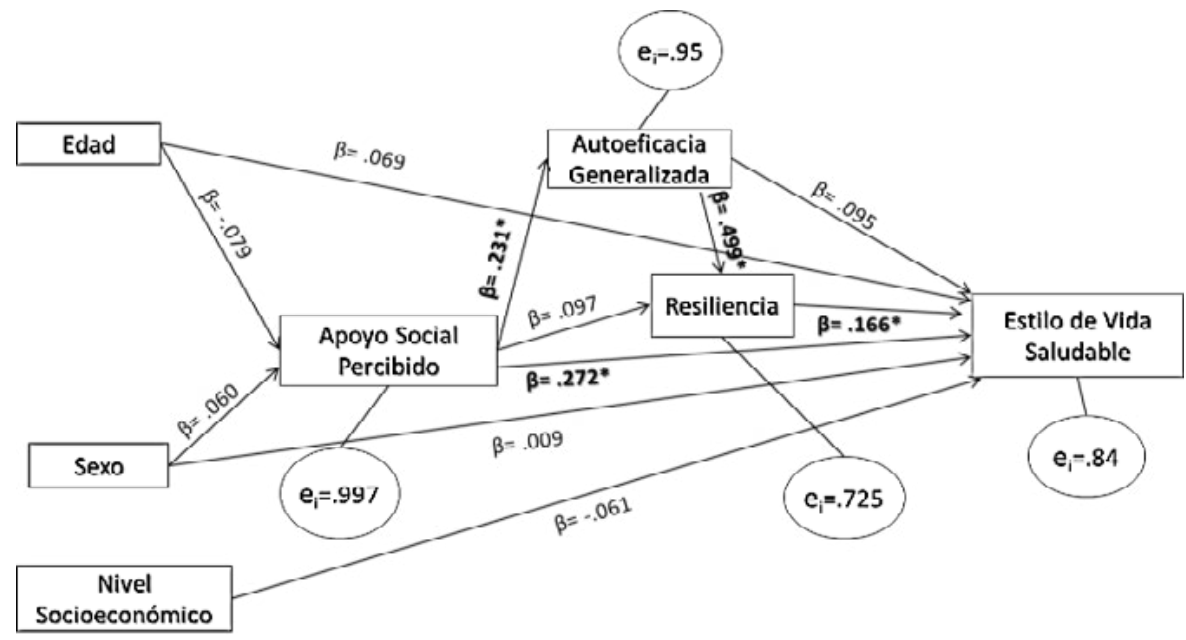

Nota: $p<0,05$

Figura 1. Diagrama de ruta obtenido donde se observa la influencia directa e indirecta de las variables predictoras sobre los estilos de vida saludable de los pacientes con riesgo cardiovascular.

factores protectores como la resiliencia, la autoeficacia generalizada y el apoyo social percibido sobre el estilo de vida saludable, en pacientes con riesgo cardiovascular. Además, busca establecer las posibles relaciones directas e indirectas entre estas, que se enmarcan en el área de la psicología de la salud.

Para poder determinar en qué nivel se encuentra la muestra respecto a cada variable, la primera parte de la discusión se enmarca alrededor de los puntajes obtenidos en cada escala utilizada y cómo esto coincide o no con otros estudios. Al respecto, la muestra estudiada, con una edad promedio de 51 años, presenta un nivel medio de estilo de vida saludable, tienen unos hábitos de vida moderados dirigidos a preservar la salud y prevenir las complicaciones cardiovasculares. Estos resultados concuerdan con los estudios de López et al. (2014b) y Rojas et al. (2016) realizados en población venezolana, donde se encontraron factores de riesgo y de protección, enmarcados estos últimos en conductas saludables moderadas-altas para el sistema cardiovascular, como alimentación, ejercicio físico, consumo de tabaco y alcohol.
Estos resultados permiten fortalecer el hecho de que no solo es indispensable la disminución del riesgo en la población, que da lugar al enfoque patogénico, sino además fortalecer el estilo de vida saludable para evitar gastos innecesarios que acarrean los costos médicos, orientándose, además, a la modificación de conductas para preservar la salud; este es uno de los objetivos fundamentales de la psicología de la salud (Becoña, Vásquez, \& Oblitas, 2004). Como resultado de este enfoque, el objetivo principal sería un modelo salugénico que integraría además del bienestar, las fortalezas o rasgos positivos en las personas; este último es una de las variables más importantes: la resiliencia.

De esta forma, la muestra estudiada reporta características moderadas de resiliencia, lo cual es necesario para llevar a cabo conductas saludables. Esto coincide con los resultados obtenidos por González, Santos, Paiva, Aparecida, Aparecida y Alcalá (2016), quienes reportaron que pacientes con enfermedades cardiovasculares de etiología clínica o quirúrgica presentaban características resilientes moderadas que les permitirían superar la situación de salud, por lo que resulta necesario 
desarrollarla en cualquier tipo de pacientes, indistintamente de la gravedad de la situación. Este constructo: resiliencia, contribuye al desarrollo saludable en presencia de factores de riesgo como la hipertensión arterial, que funciona como un mecanismo superador de problemas adversos y estresantes.

Otra variable importante es la autoeficacia generalizada, la cual permite explicar el cambio conductual en la preservación de la salud, observándose una alta prevalencia en la muestra, lo cual contribuye en gran medida al desarrollo de un estilo de vida saludable, es decir que al presentar las personas altos índices de confianza en las propias capacidades, manejan de forma adecuada los distintos estresores vitales (Canales, \& Barra, 2014; Correa, Ríos, Herrera, Busto, \& Rojas, 2015), por lo que es razonable pensar que a mayor expectativa de autoeficacia, mejor es el estilo de vida. Estos resultados coinciden con los estudios realizados por Rueda y Pérez (2004) y Campos y Pérez (2007), en los que se identificó un nivel alto de la creencia de autoeficacia en pacientes en el área de la salud; es en este contexto que se toma como variable primordial.

Esto abre una puerta a la posibilidad de que, si este constructo ha sido utilizado en conductas tan diversas en el área de la salud, sería una propuesta que funcionaría como un elemento catalizador de conductas saludables, para influir sobre el modo de pensar, sentir y actuar de la muestra seleccionada, de manera que, si alguien desarrollara esta capacidad en su máxima expresión, estaría contribuyendo a su salud (Correa et al., 2015).

Sin embargo, la autoeficacia no es la única variable que se destaca en los resultados. Con respecto al apoyo social percibido, se observó que la muestra del estudio percibe un apoyo social alto, es decir valoran subjetivamente estar rodeados por personas de confianza con las que pueden contar en momentos de crisis. Este fue un hallazgo similar al reportado en la investigación realizada por Rosa, Martin y Bayarre (2007), donde la mayoría de los pacientes hipertensos presentaron altos puntajes de apoyo social, que se relacionaron en gran medida a la adherencia terapéutica, reduciendo no solo riesgos asociados a ella, sino evitando caer en conductas poco saludables, además de tener una función reguladora de la presión sanguínea.

Una vez definidas las características de la muestra respecto a cada variable, se procederá a enmarcar las relaciones múltiples planteadas en el diagrama de ruta de acuerdo con cada hipótesis.

De esta manera, se encontró que el estilo de vida saludable está influenciado significativamente por el apoyo social percibido y la resiliencia. Esto quiere decir que aquellos participantes que reportan tener mayor resiliencia y percibir más el apoyo social de personas que los rodea, se conducirían a un mejor estilo de vida saludable. Estos resultados coinciden con los estudios reportados por Tawalbeh, Tubaishat, Batiha, Al-Azzam y AlBashtawy (2015), Acosta y Sánchez (2009) y Costa, Barontini, Forcada, Carrizo y Almada (2010), en contraste con Marín y Rodríguez (2001), quienes reportaron resultados diferentes en cuanto al apoyo social percibido.

Respecto al apoyo social, los resultados concuerdan con Tawalbeh et al. (2015), quienes refieren que el apoyo social percibido es un factor protector de la salud, destacando la importancia para reducir riesgos asociados a ella, a través del estilo de vida.

Sin embargo, Marín y Rodríguez (2001) refieren que el tipo de apoyo que más influye sobre el estilo de vida es el de la familia, lo cual es contrario a esta investigación, donde se reporta mayor influencia del apoyo de amigos sobre el estilo de vida, un elemento que es importante tomar en cuenta.

La segunda variable con mayor predicción del estilo de vida saludable es la resiliencia, el cual se refiere a la capacidad del ser humano para hacer frente a las adversidades, observándose que a pesar de ser baja la relación, es significativa para el desarrollo de conductas dirigidas a preservar la salud; esto coincide con Acosta y Sánchez 
(2009), Antonio et al. (2015) y Zurita, Espejo, Cofré, Martínez, Castro y Chacón (2016), quienes revelan que a mayores niveles de resiliencia, mejor es el hábito del sueño, la alimentación, la actividad física, las conductas preventivas, el chequeo médico y menor consumo de drogas lícitas e ilícitas, y por lo tanto menor el riesgo a desarrollar hipertensión arterial.

No obstante, a pesar de haber encontrado relaciones significativas entre la resiliencia y el apoyo social percibido con el estilo de vida saludable, se observó que la fuerza de la predicción varía según la dimensión específica de cada variable y el número de variables involucradas. El apoyo de amigos es el más significativo.

Además, al realizar las relaciones de cada variable de forma simple y no múltiple, se encontró que jerárquicamente según el nivel de importancia, el apoyo social percibido y la resiliencia no son las únicas variables que influyen significativamente sobre el estilo de vida saludable; la autoeficacia y el nivel socioeconómico son otras que las predicen. Es decir que, al plantear la interacción de todas estas variables al mismo tiempo sobre el estilo de vida, algunas se ven neutralizadas por otras, disminuyendo su nivel de influencia sobre el estilo de vida saludable.

Esto concuerda con Carpi, González, Zurriaga, Marzo y Buunk (2009), quienes estudiaron las diferencias significativas entre la autoeficacia y la percepción de control sobre las conductas preventivas de la enfermedad cardiovascular. Concluyeron que la autoeficacia influye significativamente sobre la conducta preventiva más que la percepción de control, por lo que esta variable se relaciona profundamente con el estilo de vida, cuando no se presentan otras variables que puedan minimizar su efecto directo.

Por su parte, Rosvall, Engstrom, Berglund y Hegblad (2008) afirman que el nivel socioeconómico influye de forma significativa en el tipo de conducta que se realiza, afectando positiva o negativamente la salud, es decir, a mayor nivel socioeconómico, mayor es el estilo de vida saludable. Sin embargo, este estudio tuvo una diferencia en la dirección de la relación, mientras que en los resultados arrojados por Rosvall et al. (2008) la dirección de la relación fue positiva, en la presente investigación ocurrió lo contrario, es decir, a menor nivel socioeconómico mejor fue el estilo de vida saludable. Además, se observa que cuando están presentes otras variables, se contrarresta su efecto directo sobre las conductas preventivas, lo que puede explicar que el nivel socioeconómico en las relaciones múltiples no haya resultado significativo.

Por último, en cuanto a la edad y el sexo se evidencia que estas no presentan relaciones significativas con el estilo de vida saludable. Esto puede explicarse porque en su mayoría prevalece la edad mayor a 50, lo cual limita verificar algunas diferencias en los rangos de edad. Asimismo, ocurre con el sexo, el cual resultó no significativo respecto al estilo de vida saludable, resultado que no coinciden con lo propuesto por Tawalbeh et al. (2015), quienes reportaron que existe una diferencia en el estilo de vida de acuerdo con el sexo, es decir, las mujeres presentaban mejor estilo de vida en relación con los hombres.

Si bien es cierto que las relaciones planteadas anteriormente representan la ruta principal o directa hacia el estilo de vida saludable, existen otras que interactúan hasta llegar a la conducta saludable, que funcionan como variables moderadoras.

En cuanto a la variable de resiliencia, en la presente investigación se evidencia que a pesar de que la autoeficacia no es un predictor significativo en las relaciones múltiples planteadas sobre el estilo de vida saludable, sí lo es para el desarrollo de la resiliencia, es decir que a mayor autoeficacia, mayores son las características resilientes de la persona. De manera que coincide con lo expuesto por García et al. (2016), quienes señalan que la resiliencia no es un recurso que se forma de la nada, sino que lo hace en la interacción con otras variables, entre las que se destaca la autoeficacia. 
Esto permite observar que la autoeficacia, si bien no tiene un efecto directo sobre el estilo de vida saludable en interacción con otras, sí lo tiene de forma indirecta sobre la resiliencia. Es decir, esta última funciona como una variable moderadora entre la autoeficacia y el estilo de vida saludable.

Sin embargo, a pesar de encontrarse una relación positiva significativa de la resiliencia con la autoeficacia, en el caso del apoyo social percibido no ocurre lo mismo, se observa que esta última no influye significativamente sobre la resiliencia, lo cual contradice lo que refieren Rodríguez, Ramos, Ros y Fernández (2015) y Leiva, Pineda y Encina (2013), quienes destacan que la percepción de apoyo social, familiar y de amigos funciona como factor protector de la esta.

Por otra parte, de acuerdo con Acuña y González (2010) la variable autoeficacia no se genera de la nada, se desarrolla en interacción con el ambiente social. Estos recursos van apareciendo y contribuyen al desarrollo de un adecuado sentido de autoeficacia. Es en este sentido que el apoyo social percibido entra en vigor como predictor importante de la autoeficacia. Mientras que la autoeficacia afecta de forma directa la resiliencia, el apoyo social afecta la autoeficacia, la cual resulta como variable moderadora entre ellas dos. Esto concuerda con lo propuesto por Avendaño y Barra (2008), quienes afirman que la autoeficacia se desarrolla en interacción con otras variables, entre ellas la percepción de apoyo social, dado que permite adquirir conocimientos acerca de sí mismo y del mundo, además de desarrollar competencias que contribuyen al sentido de confianza en sí mismo.

Así como el apoyo social percibido afecta significativamente a la autoeficacia, generalmente se plantea que existen variables sociodemográficas que influyen sobre ella. Así pues, como última ruta planteada, se observa que la edad y el sexo no son predictores importantes con respecto al apoyo social percibido, lo cual concuerda con Fontibón, Acosta, Valdelamar, Valdelamar y Sánchez (2015), quienes concluyeron que la edad y el sexo no tienen relación significativa ni un efecto directo sobre el apoyo social percibido. Esto quiere decir, que el desarrollo de una variable de apoyo social se produce indistintamente de la edad y el sexo.

A pesar de sus limitaciones, no se puede negar su relación indirecta sobre la salud, de manera que al menos con la presencia de una persona significativa, aumentaría la creencia de autoeficacia, la resiliencia y por lo tanto se facilitaría el estilo de vida saludable. Por ello, esta variable de apoyo social se enmarca en un ámbito multifactorial de la conducta.

En síntesis, se encontró que aquellas personas con apoyo social percibido de amigos, niveles altos de autoeficacia, menor nivel socioeconómico y características resilientes realizan conductas más saludables. Esta última variable es influenciada de forma directa e indirecta por las otras. De esta forma, los hallazgos de la investigación permiten verificar, en parte, el modelo planteado. Lo que brinda un aporte significativo en el marco de la psicología de la salud, específicamente en el área de la psicocardiología: permite observar algunas variables enmarcadas en el auge de la psicología positiva como protectores de la salud, más allá de la simple disminución del riesgo. Así, se pueden considerar futuras investigaciones que amplíen el campo de las variables positivas, además de incluirlas dentro de la prevención y tratamiento durante la práctica.

Cabe destacar que, aunque no se encontraron todas las relaciones directas en el modelo planteado, existen relaciones indirectas a través de otras variables como la autoeficacia y la resiliencia, que funcionan como variables moderadoras en muchos casos. De esta manera, más allá de responder a todas las preguntas, esta investigación abre la puerta a nuevos interrogantes, lo cual es igualmente significativo para las intervenciones en el área.

Con base en los resultados obtenidos en la presente investigación, se puede plantear que dentro de la población de pacientes que asisten al 
centro de salud, el nivel de estilo de vida saludable está determinado principalmente por la percepción del apoyo social y por la resiliencia, las cuales son importantes para desarrollar hábitos dirigidos a preservar la salud, especialmente los hábitos referidos al sueño y al consumo de droga.

A pesar de los hallazgos de que el estilo de vida se ve influenciado por variables psicológicas, aún en la actualidad se le da mayor importancia al enfoque biomédico, esto hace necesario redefinir objetivos de intervención donde se tomen en cuenta el desarrollo de las potencialidades o variables psicológicas que contribuyan no solo a prevenir la enfermedad, sino también a preservar la salud y evitar aumentar el riesgo de una enfermedad cardiovascular e incluso otras complicaciones mayores.

Con la puesta en práctica de los elementos psicológicos anteriores pueden equilibrarse los procesos de compromisos que mantenga el paciente con su propia salud, formando un ente activo en el proceso, que no solo depende de medicamentos que regulen su organismo, sino de variables que se pueden modificar. Además, conocer un posible efecto moderador que cumple la autoeficacia y la resiliencia sobre otras variables conductuales de salud permite seguir investigando a profundidad los mecanismos psicológicos involucrados en este ámbito. El estilo de vida saludable se ve influenciado no solo de forma directa, sino indirecta por otras variables, que actúan sinérgicamente entre ellas.

Así mismo, las personas que poseen elevados niveles de autoeficacia aumentan su probabilidad de presentar características resilientes, al tiempo que, si presentan una percepción mayor de apoyo social, aumenta su sentido de autoeficacia. Es decir, a mayor nivel de apoyo, mayor es la autoeficacia y la resiliencia y por lo tanto mayor el estilo de vida saludable. Se destaca así la importancia de potenciar, no solo las variables que tienen relación directa con el estilo de vida, sino las que tienen relación indirecta, pues estas son igualmente piezas clave en la preservación de la salud.
Así, se concluye que los pacientes en riesgo cardiovascular, específicamente con hipertensión arterial, mantienen un mejor estilo de vida a medida que perciben más apoyo. Mientras se sientan más capaces de llevar a cabo una conducta, aumentará el nivel de conductas saludables. Si perciben mejor apoyo social, su sentido de autoeficacia crecerá. Al aumentar el apoyo social percibido, aumentará el sentido de autoeficacia, la resiliencia y, por lo tanto, el estilo de vida saludable. Hay variables que, aunque se neutralizan bajo relaciones múltiples, mantienen relación positiva o negativa significativa cuando se relacionan directamente con otras como es el caso de la autoeficacia y el nivel socioeconómico sobre el estilo de vida. Además, cada una presenta componentes específicos que contribuyen a preservar la salud. Excluir una variable por no presentar relación significativa directa no es factible.

\section{Referencias}

Acosta, I., \& Sánchez Y. (2009). Manifestación de la resiliencia como factor de protección en enfermos crónicos terminales hospitalizados. Psicología Iberoamericana, 17(2), 24-32.

Acuña, M., \& González, A. (2010). Autoeficacia y red de apoyo social en adultos mayores. Journal of Behavior, Health \& Social Issues, 2(2), 71-81.

Angelucci, L. (2001). Influencia de las variables sociodemográficas, el apoyo social y locus de control de salud sobre la salud en empleados universitarios. (Trabajo de ascenso no publicado, Universidad Simón Bolívar, Sartenejas, Venezuela).

Angelucci, L. (2007). Análisis de ruta: conceptos básicos. Analogías del Comportamiento, $9(1)$, $31-59$

Angelucci, L., \& Cañoto, Y. (2010). Estilos de vida en los estudiantes de la escuela de psicología UCAB-Caracas. (Trabajo de grado, Universidad Católica Andrés Bello - UCAB, Venezuela). 
Antonio, M., Arrioja, G., de León, A., Velasco, L., Torres, A., Morales, F., ... Torres, M. Y. (2015). Resiliencia, conductas de salud y consumo de drogas en estudiantes universitarios. European Journal of Child Development, Education and Psychopathology, 3(2), 55-67. https://doi. org/10.30552/ejpad.v3i2.29

Arteaga, A., Collogo, R., \& Muñoz, D. (2017). Apoyo social y control metabólico en la diabetes mellitus tipo 2. Revista Cuidarte, 8(2), 1668-1676. https://doi.org/10.15649/cuidarte.v8i2.405

Avendaño, M., \& Barra, E. (2008). Autoeficacia, apoyo social y calidad de vida en adolescentes con enfermedades crónicas. Terapia Psicológica, 26(2), 165-172.

Bäbler, J., Schwarzer, R., \& Jerusalem, M. (1993). Spanish adaptation of the General Self-efficacy Scale. Recuperado de http://www.yorku. ca/faculty/academics/schwarze/spanscal.htm

Bandura, A. (1977). Self-efficacy: Toward a unifying theory of behavioral change. Psychological Review, 84, 191-215. https://doi. org/10.1037//0033-295x.84.2.191

Becoña, E., Vásquez, F., \& Oblitas, L. (2004). Promoción de los estilos de vida saludables. Investigación en Detalle Número, (5), 1-39.

Blanco, J. (2011). Emociones "positivas" y salud cardiovascular. En Y. Cañoto, G. Peña, \& G. Yaber (eds.), Tópicos en psicología de la salud (pp. 123-151). Caracas: Universidad Católica Andrés Bello.

Canales, S., \& Barra, E. (2014). Autoeficacia, apoyo social y adherencia al tratamiento en adultos con diabetes mellitus tipo II. Psicología y Salud, 24(2), 167-173.

Campos, S., \& Pérez, J. (2007). Autoeficacia y conflictos decisional frente a la disminución del peso corporal en mujeres. Revista Chilena de Nutrición, 34(3), 1-17.

Carratalá, A., \& Peña, C. (2009). Resiliencia en familiares acompañantes de pacientes oncológicos. (Trabajo de grado, Universidad Metropolitana, Caracas, Venezuela).
Carpi, A., González, P., Zurriaga, R., Marzo, J., \& Buunk, A. (2009). Autoeficacia y percepción de control en la prevención de la enfermedad cardiovascular. Universitas Psychologica, 9(2), 423-432.

Cid, P., Orellana, A., \& Barriga, O. (2010). Validación de la escala de autoeficacia general en Chile. Revista Médica Chile, 138, 551-557.

Córdoba, R., Camarelles, F., Muñoz, E., Gómez, J., Díaz, D., Ramírez, J., López, A., \& Cabezas, C. (2014). Recomendaciones sobre los estilos de vida. Atención Primaria, 46(Supl. 4) 16-23.

Correa, A., Ríos, M., Herrera, A., Bustos, C., \& Rojas, G. (2015). Autoeficacia en salud oral: concepto, evaluación y tratamiento. Avances en Odontoestomatología, 31(5), 487-495.

Costa, S., Barontini, M., Forcada, P., Carrizo, P., \& Almada, L. (2010). Psychosocial stress and low resilience: A risk factor for hypertension. Revista Argentina de Cardiología, 78(5), 425-431.

Cuadra, A., Medina, E., \& Salazar, K. (2016). Relación de bienestar psicológico, apoyo social, estado de salud física y mental con calidad de vida en adultos mayores de la ciudad de Arica. Limite, Revista Interdisciplinaria de Filosofia y Psicología, 11(35), 56-67.

Domínguez, R., \& Ibarra, E. (2017). La psicología positiva: un nuevo enfoque para el estudio de la felicidad. Razón y Palabra, 21(1), 660-679.

Escobar, P., \& Mariño, R. (2016). Factores de riesgo de infarto agudo del miocardio en pacientes con diagnóstico de hipertensión arterial. Multimed, 20(5), 129-143.

Fontibón, L. F., Acosta, L., Valdelamar, J., Valdelamar, A., \& Sánchez, R. (2015). Asociación entre variables sociodemográficas, clínicas y calidad de vida con el apoyo social percibido en pacientes colombianos con cáncer. Avances en Psicología Latinoamericana, 33(3), 387-395. Doi: dx.doi.org/10.12804/apl33.03.2015.02

García, J., García, A., López, C., \& Dias, P. (2016). Conceptualización teórica de la resiliencia 
psicosocial y su relación con la salud. Salud y Drogas, 16(1), 59-68.

Gardner, R. (2003). Estadística para Psicología usando SPSS para Windows. México: Prentice Hall.

Gómez, A. de L., Arrioja, G., Antonio, M., Velasco, L., Torres, A., Adrián, F., ... Torres, M. Y. (2016). Conducta de ejercicio y consumo de drogas en estudiantes universitarios. European Journal of Child Development, Education and Psychopathology, 4(1), 5-16. https://doi. org/10.30552/ejpad.v4i1.31

González, N., \& Estévez, A. (2017). El apoyo social percibido moderador entre el uso problemático de internet y la sintomatología depresiva en jóvenes adultos. Health and Addictions, 17(1), 53-62.

González, R., Llapur, R., Díaz, M., Illa, M., Yee, E., \& Pérez, D. (2015). Estilos de vida, hipertensión arterial y obesidad. Revista Cubana de Pediatría, 87(3), 273-284.

González, I., Santos, E., Paiva, L., Aparecida, L., Aparecida, R., \& Alcalá, D. (2016). Anxiety, depression, resilience and self-esteem in individuals with cardiovascular diseases. Revista Latino-Americana de Enfermagem, 24, e2836.

Guerrero, J. (2014). Resiliencia sexual en adolescentes: una revisión desde la psicología positiva. Realitas, Revista de Ciencias Sociales, Humanas y Artes, 2(1), 45-50.

Guerrero, J., \& Sánchez, J. (2015). Factores protectores, estilos de vida saludable y riesgo cardiovascular. Psicología y Salud, 25(1), 57-71.

Howard, S., Creaven, A., Hughes, B., O’leary, E., \& James, J. (2017). Perceived social support predicts lower cardiovascular reactivity to stress in older adults. Biological Psychology, 125, 70-75.

Jung, S., Jhee, J., Yoon, C., Park, J., Han, S., Kang, S., \& Yoo, T. (2016). The association between perceived social support with blood pressure and clinical outcome in incident hemodialysis patients. Journal of Hypertension, 34(supl. 1), e314.
Kerlinger, F., \& Lee, H. (2002). Investigación del comportamiento: métodos de investigación en ciencias sociales. México: McGraw-Hill Interamericana.

Leiva, L., Pineda, M., \& Encina, Y. (2013). Autoestima y apoyo social como predictores de la resiliencia en un grupo de adolescentes en vulnerabilidad social. Revista de Psicología Universidad de Chile, 22(2), 111-123.

Lira, M.A. (2015). Impacto de la hipertensión arterial como factor de riesgo cardiovascular. Revista Clínica Médica Condes, 26(2), 156-163.

López, R., Hurtado, D., López, L., Castillo, E., Martínez, J., Amaro, J., Rincón, R., \& Soto, L. (2014a). Factores de riesgo cardiovascular y estilo de vida de la Gran Maracaibo, parte II. Avances Cardiológicos, 34(2), 114-118.

López, R., Hurtado, D., López, L., Martínez, J., Chazzin, G., Castillo, E., \& Márquez, J. (2014b). Una aproximación a conocer la prevalencia de hipertensión arterial, factores de riesgo cardiovascular y estilo de vida en Venezuela. Avances Cardiológicos, 34(2), 128-134.

Luthar, S., Cicchetti, D., \& Becker, B. (2000). Research on resilience: response to commentaries. Child Development, 71(3), 573-575.

Marín, F., \& Rodríguez, M. (2001). Apoyo familiar en el apego al tratamiento de la hipertensión arterial esencial. Revista Salud Pública de México, 43(4), 336-339.

Mariñelarena, L. (2016). Recepción de la psicología positiva en la Argentina: un estudio socio-bibliométrico de artículos de revistas científicas. Revista Argentina de Ciencias del Comportamiento, 8(1), 27-42.

Méndez, H. (1994). Sociedad y estratificación. Método de Graffar-Méndez Castellano. Caracas: Fundacredesa.

Miao, L., Feng, J., Wu, L., Zhang, S., Ge, Z., \& Pan, Y. (2015). The mediating role of general self-efficacy in the association between perceived social support and oral health-related quality of life after initial periodontal therapy. BCM Oral Health, 16(68), 1-9. 
Morales, G., Guillen, F., Muñoz, S., Belmar, C., Schifferli, I., Muñoz, A., \& Soto, A. (2017). Factores de riesgo cardiovascular en universitarios de primer y tercer año. Revista médica de Chile, 145(3), 299-308. https://doi.org/10.4067/ S0034-98872017000300003

Novoa, C., \& Barra, E. (2015). Influencia del apoyo social percibido y los factores de personalidad en la satisfacción vital de estudiantes universitarios. Terapia Psicológica, 33(3), 239-245.

Pacheco, A., Suárez, L., \& Angelucci, L. (1998). Diagrama de ruta del efecto de los factores sociodemográficos, depresión y apoyo social sobre la salud percibida en estudiantes universitarios. Suma Psicológica, 5(2), 149-191.

Park, N., Peterson, C., \& Sun, J. (2013). La psicología positiva: investigación y aplicaciones. Terapia Psicológica, 31(1), 11-19.

Reigal, R. E., Videra, A., \& Gil, J. (2014). Practica física, autoeficacia general y satisfacción vital en la adolescencia. Revista Internacional de Medicina y Ciencias de la Actividad Física y del Deporte, 14(55), 561-576.

Rodríguez, T. (2016). Contribuciones al desarrollo de la Psicocardiología en Cuba, como rama de la psicología de la salud. Revista Finlay, 6(3), 221-235.

Rodríguez, A., Ramos, E., Ros, I., \& Fernández, A. (2015). Relaciones de la resiliencia con el autoconcepto y el apoyo social percibido en una muestra de adolescentes. Acción Psicológica, $12(2), 1-14$.

Rojas, M., Rosales, Y., Guerrero, N., Morillo, J., Añez, R., Bermúdez, V., \& Rojas, J. (2016). Comportamiento epidemiológico de la hipertensión arterial en individuos adultos del municipio San Cristóbal del estado Táchira-Venezuela. Revista Latinoamericana de Hipertensión, 11(1), 1-11.

Rosa, Y., Martín, L., \& Bayarre, H. (2007). Adherencia terapéutica y factores psicosociales en pacientes hipertensos. Revista Cubana Medicina General Integral, 23, 1-5.
Rosvall, M., Engström, G., Berglund, G., \& Hegblad, B. (2008). C-reactive protein, established risk factors, and social inequalities in cardiovascular disease - The significance of absolute versus relative measures of disease. BMC Public Health, 8, 1456-1466.

Rueda, B., \& Pérez, A. (2004), Personalidad y percepción de autoeficacia: influencia sobre el bienestar y el afrontamiento de los problemas. Revista de Psicopatología y Psicología Clínica, 9(3), 205-219.

Salazar, I., \& Arrivillaga, M. (2004) El consumo de tabaco, alcohol y otras drogas como parte del estilo de vida de jóvenes universitarios. Revista Colombiana de Psicología, 13, 74-89.

Seligman, M. (1999). The president's address. APA 1998. Annual Report. American Psychologist, 54, 559-562.

Tawalbeh, L., Tubaishat, A., Batiha, A., Al-Azzam, M., \& AlBashtawy, M. (2015). The relationship between social support and adherence to healthy lifestyle among patients with coronary artery disease in the North of Jordan. Clinical Nursing Research, 24(2) 121-138.

Uribe, A.F. (2016). Salud sexual, apoyo social y funcionamiento familiar en universitarios. Revista Virtual Universidad Católica del Norte, 49, 206-229. Recuperado de http://revistavirtual.ucn.edu.co/index.php/RevistaUCN/article/ view/805/1325

Valdéz, S., García, F., Maldonado-Araque, C., Goday, A., Calle-Pascual, A., Soriguer, F., ... Rojo-Martínez, G. (2014). Prevalencia de obesidad, diabetes mellitus y otros factores de riesgo cardiovascular en Andalucía. Comparación con datos de prevalencia nacionales. Estudio Di@bet.es. Revista Española de Cardiología. https://doi.org/10.1016/j.recesp.2013.09.031 Vázquez, C., Hervás, G., Rahona, J., \& Gómez, D. (2009). Bienestar psicológico y salud: aportaciones desde la psicología positiva. Anuario de Psicología Clínica y de la Salud, 5, 15-28. 
Veliz, L., Mendoza, S., \& Barriga, O. (2015). Adherencia terapéutica y control de los factores de riesgo cardiovascular en usuarios de atención primaria. Enfermería Universitaria, 12(1), 3-11. Venezuela, Ministerio del Poder Popular para la Salud. (2014). Anuario de mortalidad 2012. Caracas: MPPS.
Zurita, F., Espejo, T., Cofré, C., Martínez, A., Castro, M., \& Chacón, R. (2016). Influencia de la actividad física sobre la resiliencia en adultos con dolor de hombro. Spork TK: Revista Euroamericana de Ciencias del Deporte, 5(2), 53-58. 